\title{
Molecular Modeling and experimental investigation of hydrolytically degradable polymeric biomaterials
}

\author{
D. Hofmann ${ }^{1,{ }^{*}}$, M. Entrialgo ${ }^{1,2}$, J. Reiche $^{3}$, K. Kratz $^{1,4}$ and A. Lendlein ${ }^{1,4}$ \\ ${ }^{1}$ Center for Biomaterial Development, Institute of Polymer Research \\ GKSS Research Center, Kantstrasse 55, D-14513, Teltow (Germany) \\ 2 present address: Accelrys Inc., 334 Cambridge Science Park, Cambridge, UK \\ ${ }^{3}$ University of Potsdam, Institute of Physics and Astronomy, \\ Karl-Liebknecht-Strasse 24-25,D-14476 Potsdam-Golm, Germany \\ ${ }^{4}$ Berlin Brandenburg Centre for Regenerative Therapies (BCRT), Campus Virchow-Klinikum, \\ Berlin, Germany \\ "dieter.hofmann@gkss.de
}

Keywords: polymers, biomaterial hydrolytic degradation, molecular modeling, Langmuir monolayer

\begin{abstract}
Biodegradable polymers are applied in temporary implants, such as surgical sutures and controlled drug delivery systems. They are also of relevance in biomaterial-based Regenerative Therapies, where they provide a temporary substitute of the extra-cellular matrix. A major limitation of established degradable implant materials is the fact, that their degradation behavior can not be reliably predicted applying existing experimental methodologies. Therefore a knowledge-based approach is clearly needed to overcome this problem and to enable the tailored design of biodegradable polymers. Here we describe two methods, which can be applied in this approach: molecular modeling combining atomistic bulk and interface models with quantum chemical studies and experimental investigations of macromolecule degradation in Langmuir monolayers. The polymers utilized to exemplarily illustrate the concepts are aliphatic (co)polyesters [e.g. poly $(\varepsilon-$ caprolactone) (PCL), polyglycolide (PGA), poly(rac-lactide) (PDLLA), poly[(rac-lactide)-coglycolide] (PLGA)] and copoly(ether)esteruretanes as multiblock copolymers. The molecular modeling approach permits to efficiently investigate the influence of micro-structural properties like free volume distribution, cohesive energy density and concentration of polar functional groups on the bulk water uptake as one constituent part of hydrolytic degradation. The Langmuir monolayer investigations on polymer degradation on the other hand yield the dynamics of bond splitting during degradation within hours separately from time consuming diffusion processes, which may take months in bulk samples.
\end{abstract}

\section{Introduction}

Biodegradable polymers, like aliphatic poly( $\alpha$-hydroxy esters) have found several application as implant materials over the last decades. Surgical sutures, fracture and craniomaxillofacial fixations and materials in controlled drug delivery systems are prominent examples [1-5]. In biomaterialbased Regenerative Therapies such as tissue engineering approaches or stimulation of endogenous regeneration degradable polymers can be applied as temporary substitutes for the extracellular matrix (ECM) [6,7]. Each biomedical application requires a specific combination of biomaterial properties and function. Especially the elasticity and degradation rate should be adjustable. An example for a polymer system allowing the tailoring of such properties in a wide range by only small changes on the molecular level are multiblock copolymers, which were obtained by cocondensation of two different macrodiols with a low molecular weight diisocyanate $[8,9,10]$. The degradation behavior can also be influenced by the polymer architecture. The elasticity of co- 
polymer based polymer networks increases during hydrolytic degradation and abrupt changes, e.g. loss of mechanical properties leading to brittleness, can be avoided [11].

Despite this positive development there is still a considerable uncertainty regarding the predictability of the degradation behavior of the respective degrading materials, most notably concerning the decay of mechanical properties with increasing degradation time. Large differences were found between rates determined by in vitro experiments in buffer solutions or cell culture media and by in vivo studies [12,13]. The fact that systematic experimental studies on the degradation of bulk polymers are very time-consuming forms a particular additional methodical obstacle for the improvement of the described situation. Here the below described combined knowledge-based approach can be useful, which is composed of two key elements. The first one is connected with the utilization of molecular modeling methodology, which here is used on the level of Newtonian mechanics to efficiently (i.e. faster than in experimental degradation studies on bulk samples) describe important features of the water uptake in bulk degrading polymers [14-16]. The second one concerns the direct determination of the dynamics of critical bond splitting (i.e. avoiding lengthy bulk diffusion processes) via creation of monolayers of respective polymers on an LBtrough followed by characterization of the degradation rate by monitoring the area reduction versus degradation time [17-19].

Blomqvist et al. [14] used relatively small models of about 2000 atoms to assess static structural properties for polylactic and polyglycolic acids while Entrialgo et al. [15,16] for similar polymers extended this approach towards dynamic properties and the influence of the concentration of polar groups on the swelling behavior utilizing considerably larger (6000 - 8500 atoms), i.e. more realistic, models. Efficient investigations on the dynamics of bond splitting using polymer Langmuir monolayer experiments, on the other hand, were reported e.g. in refs. [17-19] while ref. [20] describes the potential of combined modeling and LB-trough investigations for a set of homopolymers. The current paper will extend this above described approach to copolymers, thus providing additional flexibility of the predictions.

\section{Bulk Degradation Processes}

In a simplified approach hydrolytic degradation can be regarded as a combined process of water uptake, which can be described by the water permeability, and a cleavage of hydrolysable bonds which can be described as a quantum chemical effect [21]. Two basic mechanisms of degradation are typically considered. One is bulk degradation, where the water uptake is considerably faster than the splitting of hydrolysable bonds. Once the polymer specimen is completely soaked with a certain amount of water cleavage of individual bonds can occur homogeneously in the whole sample. Bulk degradation is the mostly observed predominant process. (Co)polyesters from glycolic or lactic acids are typical examples of bulk degradable polymers. The second degradation mechanism is surface degradation, where the splitting of bonds proceeds much faster than the water uptake. This leads to a degradation front proceeding from the outside of a specimen towards its inside. Certain polyanhydrides and polyorthoesters, are examples for predominantly surface-eroding systems. It has to be stressed that the two models describe extreme cases while for most polymers one of the two is just dominating.

\section{Polymers and LB-Approach}

The Langmuir monolayer degradation method provides information about chain scission processes during hydrolytic or enzymatic degradation of polymers (e.g. random chain scission or chain end scission) independent from transport-phenomena, which have not to be considered in 2D degradation studies. A monolayer of the polymer of interest is spread at the air/water interface on an aqueous subphase using a LB-trough. Different aqueous subphases can be applied for monolayer degradation experiments (e.g. variation of $\mathrm{pH}$ or addition of enzymes). Afterwards the monolayer is compressed by a movable barrier to the degradation surface pressure $\left(\pi_{\mathrm{D}}\right)$. The change of the 
occupied surface area with time A(t) (so-called degradation-isotherms) obtained at $37{ }^{\circ} \mathrm{C}$ under barostatic conditions $\left(\pi_{\mathrm{D}}=\right.$ const.) is related to a continuous release of water-soluble degradation fragments from the macromolecular surface layer to the aqueous subphase. In this work we report about the hydrolytic degradation of poly(rac-latide) PDLLA and poly[( $r a c$-lactide)-co-glycolide] with $15 \mathrm{~mol} \%$ glycolide content PLGA at different $\mathrm{pH}$, as well as the selective enzymatic degradation of poly( $\varepsilon$-caprolactone) PCL and multiblock copolymers PDC synthesized from of poly( $\varepsilon$-caprolactone)diol with an number average molecular weight of $M_{\mathrm{n}}=2,000 \mathrm{~g} \cdot \mathrm{mol}^{-1}$ and poly $\left(p\right.$-dioxanone)diol $\left(M_{\mathrm{n}}=1,500 \mathrm{~g} \cdot \mathrm{mol}^{-1}\right)$ coupled via 2,2(4),4-trimethyl-hexanediisocyanate containing various PCL contents $[17,22]$ (see Table 1).

Table 1: Number average molecular weight of the of the investigated resorbable polymers

\begin{tabular}{cc}
\hline $\begin{array}{c}\text { Polymer } \\
\text { sample ID }\end{array}$ & $\begin{array}{c}M_{\mathrm{n}} \\
\left(\mathrm{g} \cdot \mathrm{mol}^{-1}\right)\end{array}$ \\
\hline PLLA & 10000 \\
PLGA & 10700 \\
PCL & 10000 \\
PDC33* & 14000 \\
PDC50* & 19000 \\
PDC67* & 22000
\end{tabular}

*the two digit number represents the PCL wt \% according to the composition of the starting mixture

The polymers utilized for the MD-simulations were the homopolymers PGA and PLLA and a stereocopolymer, rPLA50, containing 50mol $\% \mathrm{~L}$-lactide units and $50 \mathrm{~mol} \% \mathrm{D}$-lactide units, and two copolymers with the same content of glycolide, $27 \mathrm{~mol} \%$, and $73 \mathrm{~mol} \%$ of $L$-lactide in one case (PLA73GA27) and a racemic mixture of lactide in the other case, $\mathrm{PLA}_{365} \mathrm{GA}_{27}$ (i.e. $36.5 \mathrm{~mol} \% \mathrm{~L}$ lactide $36,5 \mathrm{~mol} \% D$-lactide) with a random sequence distribution.

\section{Molecular Modeling Approach}

As already mentioned in the introduction, atomistic molecular modeling can be used to look separately on water uptake. As reported elsewhere [16] quantum chemical modeling can be utilized for a separate view on the cleavage of individual bonds. Initial bulk polymer packing cells partly including different numbers of water molecules were created utilizing the amorphous cell module of the Material Studio software of Accelrys Inc. [23]. The principal course of the polymer packing algorithm was as follows: In a first stage the repeat units of interest were built. Then chains containing the number of atoms indicated in Table 2 were created from these repeat units using the polymer builder of the Material Studio software. The resulting chains served as topological templates for the next stage of the packing algorithm, namely an initial guess filling of periodic boxes (basic volume elements) with chain segments and the intended number of water molecules under periodic boundary conditions. Details of the packing algorithm are outlined e.g. in ref. [24]. The volume of the basic cell was chosen in such a way that the - experimentally obtained macroscopic density of the respective bulk polymer (cf. Table 2) is reproduced. All initial packing models had then to be subjected to extensive equilibration procedures including molecular dynamic (MD) simulations connected with forcefield parameter scaling and stimulated annealing [24] applying the Discover module of Material Studio. All simulations were performed with the modified pcff forcefield reported by Blomqvist et al [14]. Equilibration was then followed by MD data production runs typically extending over $1 \mathrm{~ns}$ at a temperature $\mathrm{T}$ of $310 \mathrm{~K}$ and snapshots of the model geometry were saved in a respective history file for every 500 fs. These simulations were performed under NPT conditions, i.e. the atom number $\mathrm{N}$ and the pressure $\mathrm{P}$ where fixed $(\mathrm{P}=$ 
1 bar). In each case three independent models were realized. Table 2 shows the respective average model parameters after complete equilibration. It needs to be noticed that each of the mentioned models was constructed and simulated with three different water contents where experimental reference data were available, $0 \mathrm{wt} . \%, 2 \mathrm{wt} . \%$ (except PGA where $2.9 \mathrm{wt} . \%$ applied) and $7 \mathrm{wt} .-\%$ to be able to characterize the micro-structural effects at the different stages of water permeation in the bulk of the respective polymers. The study therefore comprised an overall number of 45 simulated models. While some of the results mentioned below for the homopolyesters were already discussed elsewhere $[15,16,20]$, detailed results for the copolyesters are shown and discussed in comparison with the homopolymers for the first time in the current paper.

Table 2: Basic parameters of selected simulation models (all simulation data averaged over 3 models in each case)

\begin{tabular}{lllll}
\hline Polymer & $\begin{array}{l}\text { Experimental } \\
\text { density }\left(\mathrm{g} / \mathrm{cm}^{3}\right)\end{array}$ & $\begin{array}{l}\text { Av. Density } \\
\text { in 1 bar NPT- } \\
\text { MD } \\
\left(\mathrm{g} / \mathrm{cm}^{3}\right)\end{array}$ & $\begin{array}{l}\text { Lateral model } \\
\text { dimension } \\
(\mathrm{nm})\end{array}$ & No. of atoms \\
\hline PLLA & $1.24-1.30$ & 1.23 & 4.510 & 8588 \\
rPLA $_{50}$ & $1.21-1.27$ & 1.23 & 4.081 & 6328 \\
PLA $_{73} \mathrm{GA}_{27}$ & 1.3 & 1.30 & 4.178 & 6821 \\
PLA $_{355} \mathrm{GA}_{27}$ & 1.3 & 1.29 & 4.203 & 6958 \\
PGA & $1.45-1.50$ & 1.49 & 4.082 & 6342
\end{tabular}

Utilizing the structural snapshots in the recorded history files the following model properties were calculated for all models mentioned in Table 2 considering all above described water contents: water accessible fractional free volume, mean squared displacements (MSD) which are characterizing the respective diffusion for the polymer atoms and the inserted water molecules (where available), cohesive energy densities CED and related solubility parameters $\delta=(C E D)^{1 / 2}$ for all polymer models, in each case with and without inserted water molecules. Concerning the latter data it needs to be mentioned that all interactions, i.e. van der Waals, electrostatic and hydrogen bonds were considered. In addition radial distribution functions were calculated to characterize possible water clusters and hydrogen bonds in the respective polymer models.

\section{Results and Discussion}

LB Approach. For 3D hydrolytic degradation of PDLLA or PLGA both random chain scission or chain-end scission were reported in the literature. In case of a random chain scission kinetics an exponential increase of the generation rate of water-soluble fragments can be expected, while for chain-end scission kinetics a linear increase should occur. A linear area reduction in hydrolytic monolayer degradation experiments performed at different $\mathrm{pH}$ of the aqueous subphase (as displayed in Fig. 1A) was recently reported for PDLLA and PLGA with similar molecular weight of $10,000 \mathrm{~g} \cdot \mathrm{mol}^{-1}$ which clearly confirmed the chain-end scission mechanism [18]. Here also the acid or base autocatalyses of the hydrolytic degradation kinetics becomes obvious where the PDLLA degraded considerably faster at low $(\mathrm{pH} 2)$ and high $\mathrm{pH}(\mathrm{pH} 10.5)$ in comparison to neutral $\mathrm{pH}=$ 7.2. At subphase $\mathrm{pH}=10.5$ the hydrolytic bond scission rate of PLGA was found to be significantly higher than that of PDLLA (nearly two-fold), which can be attributed to the faster hydrolysis of glycolic acid ester bonds. In further monolayer studies with end-capped and non-end-capped PDLLA almost identical degradation rates were found, which indicates that the differences in the degradation kinetics observed for 3D samples should originate from differences in the water penetration into the polymers. 


\section{Figure 1: Area reduction curves obtained for of PDLLA, PLGA, PCL and PDC in hydrolytic and enzymatic monolayer degradation experiments.}

A)

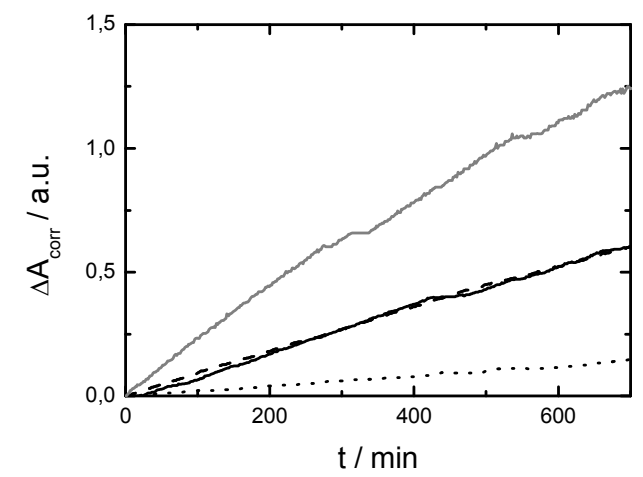

B)

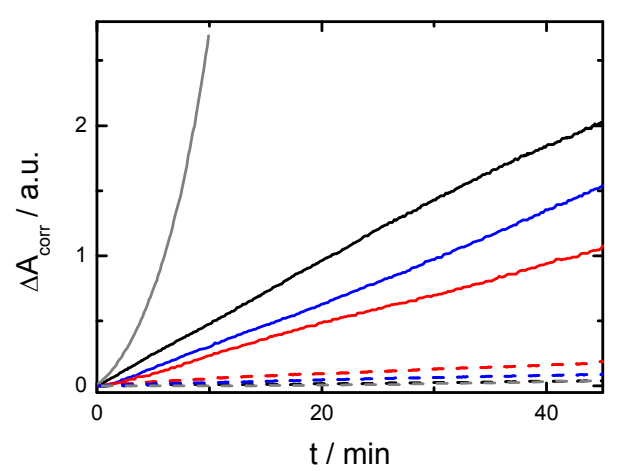

A) Influence of $\mathrm{pH}$ variation and introduction of glycolide comonomer units on the hydrolytic monolayer degradation of PDLLA ( $\mathrm{pH}=2$ : black dashed line; $\mathrm{pH}=7.2$ : black dotted line; $\mathrm{pH}=10.5$ : black solid line) and PLGA $(\mathrm{pH}=10.5$ : solid gray line). B) Influence of PCL content on the selective enzymatic degradation of PDC multiblock copolymer (33 $\mathrm{wt} \%$ : red solid line; $50 \mathrm{wt} \%$ : blue solid line; $67 \mathrm{wt} \%$ : black solid line; pure PCL: gray solid line). The dotted lines represent the results of the hydrolytic degradation experiments.

Monolayer degradation experiments can also be performed on aqueous subphases containing enzymes. For enzymatic monolayer degradation of poly( $\varepsilon$-caprolactone) containing polymers typically Pseudomonas Cepacia lipase is used [17,25]. For PCL an exponential increase of the number of dissolved degradation fragments was obtained with increasing degradation time, which indicated a random chain scission mechanism for enzymatic bond cleavage and can be described by the dynamic fragmentation theory [25].

Further more the above mentioned approach can be utilized for selective enzymatic degradation studies of PDC multiblock copolymers containing PCL segments [22]. The contribution of hydrolytic degradation of PDC as well as PCL can be neglected in the (selective) enzymatic monolayer degradation experiments. The PDC multiblock copolymers exhibit pseudolinear degradation behavior where the degradation rate was found to increase with increasing amount of PCL (see Fig. 1B). This pseudolinear degradation of PDC is in agreement with the data obtained in corresponding enzymatic degradation studies on 3D samples [19] and can be explained by dangling ends of poly( $p$-dioxanone) units which were partially immersed in the subphase.

\section{Molecular Modeling Approach.}

First considering Table 2 it should be mentioned that the quite acceptable coincidence between the experimental density values and those obtained in 1-bar NPT-MD data production runs is already one important criterion for the validation of the quality of the equilibrated packing models. Further successful validation was performed by comparing simulated and measured wide angle X-ray scattering (WAXS) patterns (not shown here) for the dry models, where these data were available experimentally. Table 3 contains important properties calculated from the simulation data 
Table 3: Properties calculated from the simulation data. (Water contents in wt.- $\%$ attached to the respective sample names. All values averaged over three independent models)

\begin{tabular}{|c|c|c|c|}
\hline Polymer model* & $\begin{array}{l}\text { Water accessible free } \\
\text { volume (rel. contents.) }\end{array}$ & $\begin{array}{c}\text { Solubility parameter } \\
\delta\left(\mathrm{J} / \mathrm{cm}^{3}\right)^{1 / 2} \\
\text { complete model }\end{array}$ & $\begin{array}{c}\text { Solubility parameter } \\
\delta\left(\mathrm{J} / \mathrm{cm}^{3}\right)^{1 / 2} \\
\text { after water removal }\end{array}$ \\
\hline PGA-0 & 0.001 & 31.9 & - \\
\hline PGA-2.9 (10 days) & 0.023 & 33.0 & 30.8 \\
\hline PGA-7 (15 days) & 0.078 & 33.9 & 28.9 \\
\hline PLLA-0 & 0.011 & 23.3 & - \\
\hline PLLA-2 (56 days) & 0.027 & 24.2 & 22.6 \\
\hline PLLA-7 (84 day) & 0.093 & 26.3 & 21.3 \\
\hline $\mathrm{rPLA}_{50}-0$ & 0.011 & 22.6 & - \\
\hline $\mathrm{rPLA}_{50}-2$ & 0.031 & 23.6 & 22.1 \\
\hline $\mathrm{rPLA}_{50^{-}} 7$ & 0.097 & 26.4 & 20.9 \\
\hline $\mathrm{PLA}_{73} \mathrm{GA}_{27}-0$ & 0.007 & 25.1 & - \\
\hline $\mathrm{PLA}_{73} \mathrm{GA}_{27}-2$ & 0.030 & 26.3 & 24.6 \\
\hline $\mathrm{PLA}_{73} \mathrm{GA}_{27} 7$ & 0.104 & 28.3 & 22.8 \\
\hline $\mathrm{PLA}_{365} \mathrm{GA}_{27}-0$ & 0.005 & 24.5 & - \\
\hline $\mathrm{PLA}_{365} \mathrm{GA}_{27}-2$ & 0.024 & 25.7 & 24.0 \\
\hline $\mathrm{PLA}_{365} \mathrm{GA}_{27} 7$ & 0.093 & 27.9 & 22.4 \\
\hline
\end{tabular}

* Respective experimental water uptake times which are available for the homo-polymers PGA [26] and PLLA [27] are provided in parentheses.

Considering the water accessible free volume column it is clearly visible that in the initial water free (dry) state PGA has considerably smaller values than PLA which seems contradictory to the experimentally observed long-term water uptake behavior, where PGA is almost one order of magnitude faster than PLLA. The finding can be however well understood from a molecular structure point of view insofar as the additional methyl side group in PLLA makes the backbone of this polymer stiffer than in PGA which typically results in a less dense chain segment packing which is leading to a larger free volume contents. The racemic copolymer $\mathrm{PLLA}_{50^{-}} 0$ which does not contain any glycolide (GA) units shows about the same water accessible free volume as PLLA, while $\mathrm{PLA}_{73} \mathrm{GA}_{27}-0$ and $\mathrm{PLA}_{365} \mathrm{GA}_{27}-0$ both containing $27 \mathrm{~mol} \%$ GA (but differing in the tacticity of the lactide units) have free volume values between PG and PLLA. This is a first indication that in the given case the GA content is more important for the water uptake than specific tacticity features.

If then the simulated development of the free volume is compared with the respective experimental water uptake data, it is clearly visible that PGA overtakes PLLA already after a relatively short time. E.g. already after 10 days PGA has a free volume content comparable to PLLA after about 56 days. This indicates a considerably higher water solubility $\mathrm{S}_{\mathrm{H} 2 \mathrm{O}}$ in PGA than in PLLA (cf. also discussion about the solubility parameters below). As in the dry case the water containing racemic copolymer models $\mathrm{rPLA}_{50^{-}} 2$ and $\mathrm{rPLA}_{50^{-}} 7$ show a swelling behavior comparable to the respective homo-polymer PLLA. The influence on the free volume of the $27 \mathrm{~mol} \%$ GA-content in the water containing $\mathrm{PLA}_{73} \mathrm{GA}_{27}$ and $\mathrm{PLA}_{365} \mathrm{GA}_{27}$ models, however seems to be negligible in difference to the situation in the dry state.

Concerning the solubility parameter some correlation with the GA content can be observed, which is qualitatively comparable for models where the calculation was performed with or without the inserted water molecules. Therefore here only the situation without the inserted water molecules shall be discussed. Pure dry PGA and PLLA have values of $31.9\left(\mathrm{~J} / \mathrm{cm}^{3}\right)^{1 / 2}$ and $23.3\left(\mathrm{~J} / \mathrm{cm}^{3}\right)^{1 / 2}$, respectively. For the models of the dry racemic $\mathrm{rPLA}_{50^{-}} 0$ a $\delta$-value of $22.6\left(\mathrm{~J} / \mathrm{cm}^{3}\right)^{1 / 2}$ was observed, which corresponds basically to the respective value of pure PLLA. The $27 \mathrm{~mol} \%$ GA containing models $\mathrm{PLA}_{73} \mathrm{GA}_{27}-0$ and $\mathrm{PLA}_{365} \mathrm{GA}_{27}-0$ on the other hand have solubility parameters between 
those of PGA and PLLA and are closer to the latter case. I.e., the higher the GA content the higher the respective $\delta$-value, while specific differences in tacticity of the respective PLLA segments do not seem not to have any significant influence. The same trends are observed for the different simulated water contents.

The $\delta$-values for the PGA models are considerably closer to the respective value for water $\left(47.9\left(\mathrm{~J} / \mathrm{cm}^{3}\right)^{1 / 2}[28]\right.$ than for the PLLA models, indicating a much higher solubility of water $\mathrm{S}_{\mathrm{H} 2 \mathrm{O}}$ in PGA than in PLLA, while the $27 \mathrm{wt} \%$ GA containing copolymers should show water solubilities between PLLA and PGA but closer to the former than to the latter. Considering the dry case, which is most relevant here also water diffusion constants can be (coarsely) estimated from the slope of the calculated water MSD-curves for the $2 \mathrm{wt} . \%$ water content models (dry models do of course not permit such calculations). The resulting $\mathrm{D}_{\mathrm{H} 2 \mathrm{O}}$-constants (in $10^{-7} \mathrm{~cm}^{2} \mathrm{~s}^{-1}$ ) are 5 for PGA, 6 for PLLA, 6 for $\mathrm{rPLA}_{50}, 7$ for $\mathrm{PLA}_{73} \mathrm{GA}_{27}$ and 4 for $\mathrm{PLA}_{365} \mathrm{GA}_{27}$. I.e. considering the standard deviations of about \pm 1 there is no systematic variation of the water diffusion constants with the respective GA content visible. Considering the fact that the water uptake can be described by a solution-diffusion model, i.e. the permeability of water $\mathrm{P}_{\mathrm{H} 2 \mathrm{O}}$ is the product of the respective diffusion constant $\mathrm{D}_{\mathrm{H} 2 \mathrm{O}}$ and the solubility $\mathrm{S}_{\mathrm{H} 2 \mathrm{O}}$ of water, these findings can improve our knowledge about the observed differences in the water uptake insofar as the differences in water solubility seem to be decisive for the observed effects. This result can in the given case easily be related back to micro-structural features. In this case the higher density of polar groups in pure PGA than in PLLA containing an additional nonpolar methyl group in the repeat unit is decisive. Since the solubility of water in a polymer is to a large extent determined by non-bonded energetic interactions of the highly polar water molecules with the functional groups of a given polymer, a higher density of polar groups in a bulk polymer will increase the respective water solubility. In the given case this leads to a similar increase in water permeability and to the related rate of water uptake.

\section{Summary}

Molecular modeling studies could be applied to obtain additional knowledge about the mechanism of water uptake in the amorphous domains of certain (PLLA, PGA and respective copolymers) hydrolytically degradable polymers, which could not be obtained by experimental means alone. From these simulations the content of the more polar GA groups is the major structural feature increasing the water solubility in the respective polymers in comparison to pure PLLA. This leads to a water uptake being the faster the higher the contents of GA groups with a maximum for pure PGA. The effect of additional GA groups on the water diffusion constants, on the other hand, seems to be negligible, i.e. the water uptake in these polymers is solubility controlled. It could also be concluded that variations in the tacticity situation in polylactic acid or in blocks of polylactic acid in copolymers with PGA do not have a significant influence on the water solubility and thus on the water uptake.

In a way complementary to the described modelling approach is was furthermore demonstrated that the Langmuir-monolayer approach for investigation of hydrolytic or enzymatic degradation is a time and material saving method (compared to conventional degradation studies using 3D samples) where information about the bond scission kinetics can be obtained independent of time-consuming diffusion processes.

In the future additional efforts are required to complement the described knowledge-based approach, like combinations of X-ray investigations with mechanical characterization and/or electron microscopy, a strategy, which was successfully applied for other polymer systems [29,30]. 


\section{Acknowledgements}

We thank the European Commission for partial financial support under grant EU-STREP "MULTIMATDESIGN- Computer aided molecular design of multifunctional materials with controlled permeability properties".

\section{References}

[1] A. Lendlein: Chemie in unserer Zeit Vol. 33 (1999), p. 279.

[2] P. A. Gunatillake and R. Adhikari: Eur. Cell. Mater. Vol. 5 (2003), p. 1.

[3] A. Goepferich: in: Handbook of Biodegradable Polymers, edited by A.J. Domb, J. Kost, D.M. Wiseman, Harwood Academic Publishers, Amsterdam 1997.

[4] D.W. Hutmacher: Biomaterials Vol. 21 (2000), p. 2529.

[5] J. C. Middleton and A. J. Tipton: Biomaterials Vol. 21 (2000), p. 2335.

[6] V.P. Shastri, A. Lendlein: Adv. Mat. Vol. 21 (2009), p. 3231.

[7] T. Weigel, G. Schinkel, A. Lendlein: Exp. Rev. Med. Dev. Vol. 3 (2006), p. 835.

[8] A. Lendlein, P. Neuenschwander, U.W. Suter: Macromol. Chem. Phys. Vol. 199 (1998), p. 2785.

[9] A. Lendlein, P. Neuenschwander, U.W. Suter: Macromol. Chem. Phys. Vol. 201 (2000), p. 1067.

[10]A. Lendlein, M. Colussi, P. Neuenschwander, U.W. Suter: Macromol. Chem. Phys. Vol. 202 (2001), p. 2702.

[11]A.T. Neffe, G. Tronci, A. Alteheld, A. Lendlein: Macromol. Chem. Phys. Vol. 211 (2010), p. 182.

[12] M. Vert, S. Li, H. Garreau, J. Mauduit and M. Boustta: Angew. Makromol. Chem. Vol. 247 (1997), p. 239.

[13] R. Chandra and R. Rustgi: Prog. Polym. Sci. Vol. 23 (1998), p. 1335.

[14] J. Blomqvist, B. Mannfors and L.-O. Pietilä: J. Mol. Struct. (Teochem) Vol. 531 (2000), p. 359.

[15] M. Entrialgo-Castano, A. Lendlein and D. Hofmann: Adv. Eng. Mater. Vol. 8 (2006), p. 434.

[16]M. Entrialgo-Castano, A. E. Salvucci, A. Lendlein and D. Hofmann: Macromol. Symp. Vol. 269 (2008), p. 47.

[17] J. Reiche, A. Kulkarni, K. Kratz and A. Lendlein: Thin Solid Films Vol. 516 (2008), p. 8821.

[18]A. Kulkarni, J. Reiche and A. Lendlein: Surf. Interface Anal. Vol. 39 (2007), p.740.

[19]A. Kulkarni, J. Reiche, J. Hartmann, K. Kratz and A. Lendlein: Eur. J. Pharm. Biopharm. Vol. 68 (2008), p. 46.

[20]D. Hofmann, M. Entrialgo-Castano, K. Kratz, and A. Lendlein: Adv. Mat. Vol. 21 (2009), p. 3237.

[21]M. Vert, S. Li and H. Garreau, J. Mauduit and M. Boustta: Angew. Makromol. Chem. Vol. 247 (1997), p. 239.

[22]H. Grablowitz, A. Lendlein: J. Mat. Chem. Vol. 17 (2007), p. 4050.

[23] Material Studio Software Package; Accelrys Inc.: San Diego, CA, 2008. 
[24] D. Hofmann, L. Fritz, J. Ulbrich, C. Schepers, M. Boehning: Macromol. Theory Simul. Vol. 9 (2000), p. 293.

[25] A. Kulkarni, J. Reiche, K. Kratz, H. Kamusewitz, I. M. Sokolov, A. Lendlein: Langmuir 2007, $23,12202$.

[26] S. Hurrel, R.E. Cameron: Biomaterials Vol. 23 (2002), p. 2401.

[27] S. Li, H. Garreau, M. Vert: J. Mater. Sci. Mater. Med. Vol. 1 (1990), p. 198.

[28] H. Mark (ed.): Encyclopedia of Polymer Science and Technology (Wiley-VCH, Germany, 2004).

[29]D. Hofmann, E. Schulz: Polymer Vol. 30 (1989), p. 1964.

[30] H.-P. Fink, D. Hofmann, H.J. Purz: Acta Polym. Vol. 41 (1990), p. 131. 